\title{
BTeV - A Dedicated B Physics Experiment at the Tevatron Collider
}

\author{
Klaus Honscheid* ${ }^{\dagger}$ \\ The Ohio State University, Columbus, Ohio \\ E-mail: 'kh@mps.ohio-state.edü
}

\begin{abstract}
Exploiting the large number of heavy quarks produced at the Tevatron collider, the $\mathrm{BTeV}$ experiment is designed to make precision measurements of Standard Model parameters and to perform an exhaustive search for physics beyond the Standard Model. Last year the BTeV collaboration has been given approval by the Fermilab director. In our presentation at the HEP 2001 conference we discussed some of the many technological challenges the $\mathrm{BTeV}$ collaboration faces designing and building the detector. High interaction rates with a very unfavorable signal to noise ratio, large radiation backgrounds and the need to minimize the amount of material in the fiducial volume requires innovative designs for every detector component.
\end{abstract}

This report will not provide a detailed discussion of the BTeV detector. As we are in an active research and development phase the interesting details will change much faster than the time scales typical for the publication of conference proceedings. Instead, we will give only a brief description of the major components of the $\mathrm{BTeV}$ spectrometer and provide links to the up-to-date information on the World Wide Web.

\section{The BTeV Experiment}

General information about $\mathrm{BTeV}$ is available on the collaboration's web site. This site can be accessed using the URL:

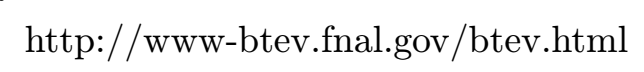

The transparencies of the presentation can be found on the conference web site:

$$
\text { http:_/_www.hep } 200 \overline{1} \text {.elte.hu/! }
$$

The transparencies can also be downloaded in pdf format from the author's web site using the URL:

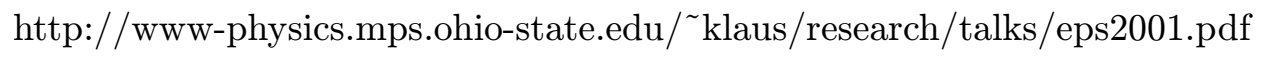

${ }^{*}$ Speaker.

${ }^{\dagger}$ On behalf of the BTeV Collaboration. 


\section{The BTeV Detector}

A schematic view of the detector is shown in Figure that used in current collider experiments. Each arm of the spectrometer covers the forward direction, $10-300 \mathrm{mrad}$, with respect to the colliding beams. This geometry allows BTeV to take advantage of the large b-production cross section at the Tevatron, a large Lorentz boost in the forward/backward direction which increases the reconstruction efficiency for $\mathrm{B}$ decays and improves the proper time resolution, as well as correlations in $b \bar{b}$ production at hadron colliders which significantly improve flavor tagging efficiencies.

Charged particle tracking and vertexing is accomplished by a combination of a silicon pixel vertex detector and a forward tracker. The pixel detector will contain approximately $3 \times 10^{7}$ rectangular pixels, each $50 \mu \mathrm{m} \times 400 \mu \mathrm{m}$, connected to a custom designed read-out circuit by a "bump bond". The pixel detector is arranged in 31 stations each consisting of two $10 \mathrm{~cm} \times 10 \mathrm{~cm}$ planes. A $12 \mathrm{~mm} \times 12 \mathrm{~mm}$ hole is cut out for the colliding beams. Details of the BTeV pixel detector and the custom designed readout electronics can be found at inttp: [ [ www-rhve.fnal.gov/.

The forward tracker consists of 4-mm diameter straw tube driftchambers and silicon strip detectors in the high occupancy areas near the beam pipe. Further details are available at http:_/_www-btev.fnal.gov/_workgroup/straws/straws.htmín

and htttp: L/www-btev.fnal.gov/workgroup/tracking/index.htmli

Particle identification over a wide momentum range will be provided by ring-imaging Cherenkov counters (RICH). Access hittp:/Lwww-btev.fnal.gov/_workgroup/pid/index.htwinl', for more information on the $\mathrm{BTeV}$ RICH detector.

The BTeV design includes an electromagnetic calorimeter for photon and neutral pion reconstruction as well as electron identification. The $\mathrm{BTeV}$ calorimeter will be built out of $\mathrm{PbWO}_{4}$ crystals, very similar to the CMS calorimeter. Detailed information on the design. performance and readout of the $\mathrm{BTeV} \mathrm{EM}$ calorimeter is available at

$$
\text { inttp:L/wwww-btev.fnal.gov/ecal } / \text { i }
$$

The BTeV Muon detector (http: L L web.hep.uiucedu dent momentum measurements for muon candidates using two $1 \mathrm{~m}$ long steel toroid magnets at $1.5 \mathrm{~T}$. The active detection planes are built out of stainless steel proportional tubes.

Of central importance to the $\mathrm{BTeV}$ physics program is the detached vertex trigger. Using information from the silicon pixel detector this trigger searches every beam crossing for detached vertices, the characteristic signature of $b$ quark decays. This first level trigger together with higher trigger levels and a high performance data acquisition system will give $\mathrm{BTeV}$ high efficiency for interesting $b$ decays combined with excellent background suppression. Further information on the trigger and data acquisition systems can be found at:

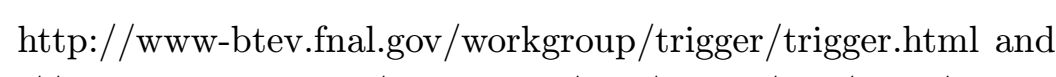
http:L/ 


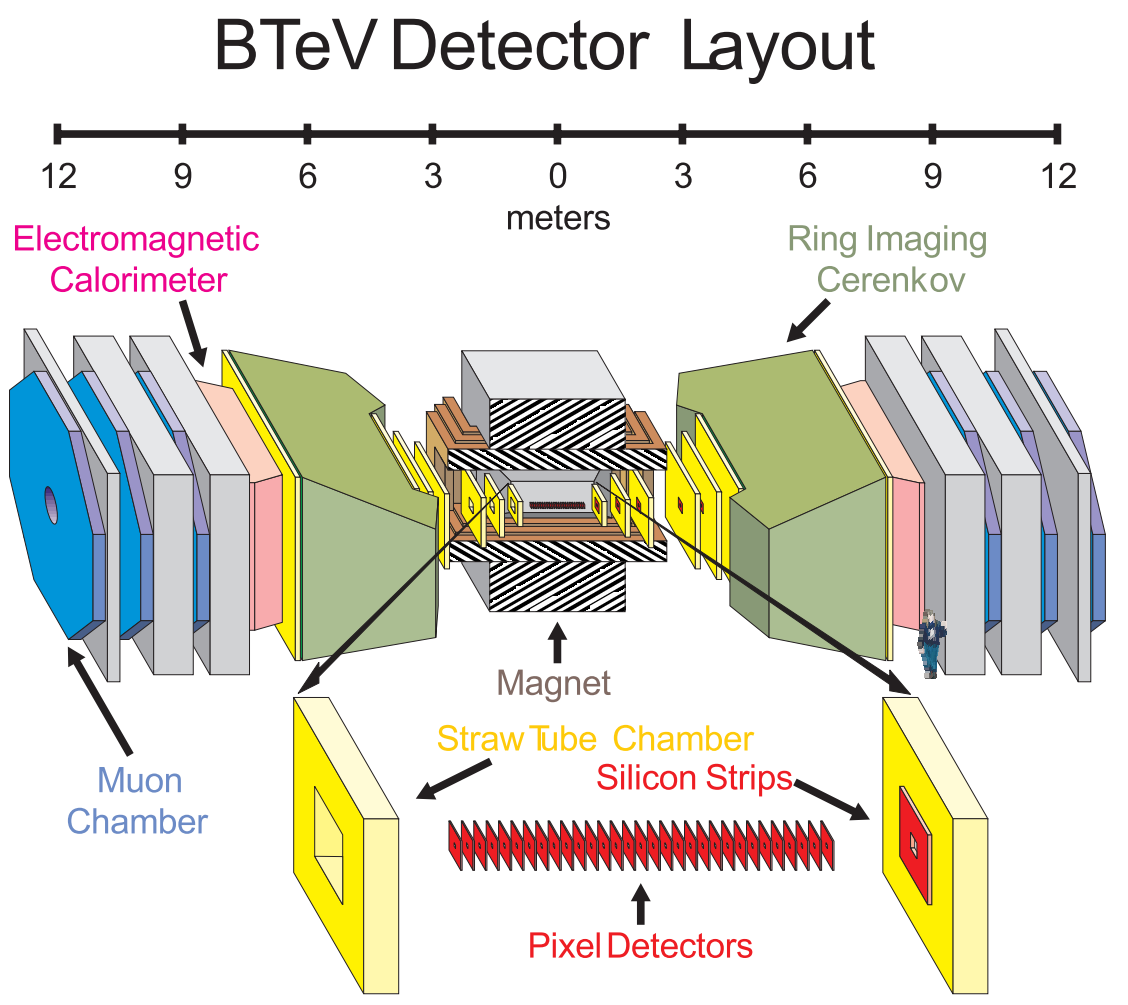

Figure 1: Schematic view of the BTeV Spectrometer. 